\title{
XMM-Newton detection of a Comptonized accretion disc in the quasar PKS 0558-504
}

\author{
P. T. O’Brien ${ }^{1}$, J. N. Reeves ${ }^{1}$, M. J. L. Turner ${ }^{1}$, K. A. Pounds ${ }^{1}$, M. Page ${ }^{2}$, M. Gliozzi ${ }^{3}$, W. Brinkmann ${ }^{3}$ \\ J. B. Stephen ${ }^{4}$, and M. Dadina ${ }^{4}$ \\ 1 X-Ray Astronomy Group, Department of Physics and Astronomy, University of Leicester, LE1 7RH, UK \\ 2 MSSL, University College London, Holmbury St. Mary, Dorking, Surrey, RH5 6NT, UK \\ 3 Max-Plank-Institut für extraterrestrische Physik, Postfach 1312, 85741, Garching, Germany \\ ${ }^{4}$ Istituto TESRE, CNR, Via Gobetti 101, 40129 Bologna, Italy
}

Received 27 September 2000 / Accepted 19 October 2000

\begin{abstract}
We present XMM-Newton observations of the bright quasar PKS 0558-504. The $0.2-10 \mathrm{keV}$ spectrum is dominated by a large, variable soft X-ray excess. The fastest flux variations imply accretion onto a Kerr black hole. The XMM-Newton data suggest the presence of a "big blue bump" in PKS 0558-504 extending from the optical band to $\sim 3 \mathrm{keV}$. The soft X-ray spectrum shows no evidence for significant absorption or emission-line features. The most likely explanation for the hot big blue bump is Comptonization by the multi-temperature corona of a thermal accretion disc running at a high accretion rate.
\end{abstract}

Key words. galaxies: active - X-rays: galaxies - accretion discs - quasars: individual: PKS 0558-504

\section{Introduction}

The most prominent feature in the broad-band spectral energy distribution of many active galactic nuclei (AGN) is the "big blue bump" (BBB), which contains a large fraction of the bolometric luminosity. In most broad-line AGN the BBB begins around $1 \mu \mathrm{m}$, rises to a peak in the ultraviolet and then declines again into the unobservable EUV (e.g. Elvis et al. 1994). The soft X-ray excesses seen in many AGN may be the high-energy tail of the BBB (e.g. Turner \& Pounds 1989; Walter et al. 1994; Laor et al. 1997). The harder $2-10 \mathrm{keV}$ X-ray spectrum can usually be quite well described by a power-law with photon-index $\Gamma \approx 1.9$. Most AGN also show an iron $\mathrm{K} \alpha$ fluorescence feature around 6-7 keV and/or a hump of emission above $10 \mathrm{keV}$ (e.g. Pounds et al. 1990), widely interpreted in terms of Compton reflection of hard Xrays by optically-thick matter probably in the form of an accretion disc (George \& Fabian 1991).

Three basic classes of model have been proposed to explain the BBB. The first class involves thermal emission from an optically thick accretion disc surrounding the central black hole in which the primary heating is by viscous dissipation within the disc itself (e.g. Shields 1979). The disc thermal spectrum may be comptonized by a hot disc corona; a process which changes the observed shape

Send offprint requests to: P. T. O'Brien,

e-mail: pto@star.le.ac.uk of the continuum, helping to explain soft X-ray emission from discs which are otherwise too cool (e.g. Czerny \& Elvis 1987). A second class of models also involves thermal emission from an accretion disc, but with irradiation by a hard X-ray power-law (perhaps arising from magnetic flares) as the primary energy source (e.g. Ross \& Fabian 1993). In these models the disc reprocesses the hard X-ray emission into thermal emission which is reradiated at the local disc temperature. Irradiated discs may have flatter radial temperature profiles, and hence different spectra, compared to discs dominated by viscous dissipation. The final class of model involves optically thin, thermal free-free emission from a hot plasma (Barvainis 1993), although single temperature models of this kind do not fit the observed continuum very well (Siemiginowska et al. 1995).

While the spectral form of the optical and UV continuum in AGN are well constrained by existing data, the soft X-ray continuum shape is often quite poorly defined for individual QSOs and therefore places only limited constraints on BBB models. These limitations have been due to the low spectral resolution, signal-to-noise ratio and/or narrow spectral bandpass provided by previous X-ray instruments, thereby making it difficult to quantify absorption/emission features which may contaminate the $\mathrm{X}$-ray spectrum and hence distort the derived continuum shape. The advent of XMM-Newton with its broad bandpass, high throughput and good spectral resolution now 


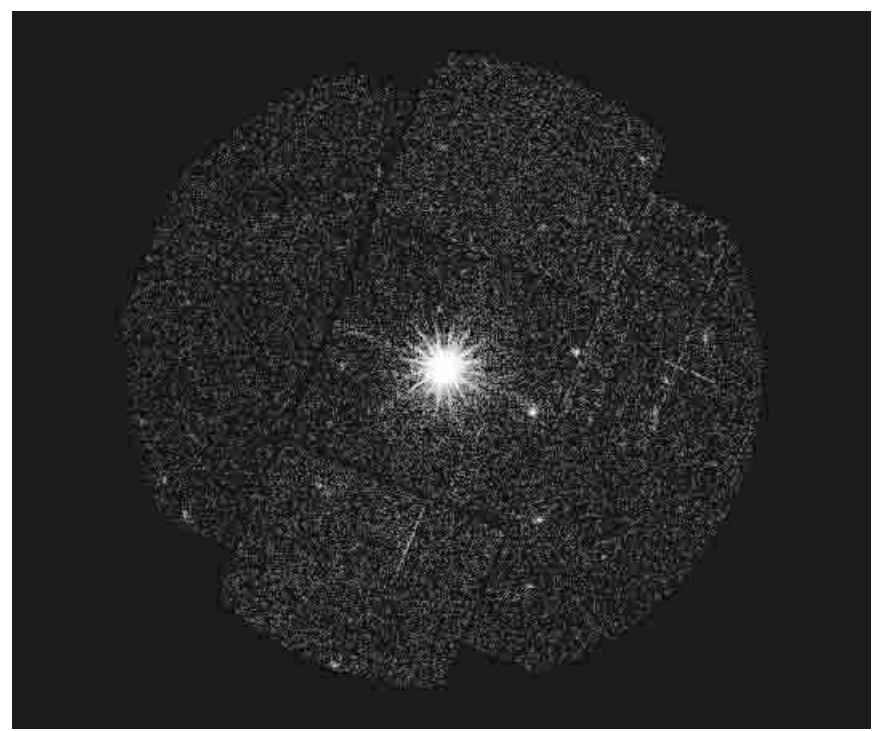

Fig. 1. A Full-Window mode EPIC MOS-1 image of PKS 0558-504 taken during XMM-Newton orbit 42. A logarithmic intensity scale has been used to show the point spread function. No other strong X-ray sources are seen within the 30 arcmin field of view

provides the opportunity to obtain high-quality X-ray spectra and hence make progress in defining the intrinsic shape of the X-ray continuum in AGN.

In this paper we present XMM-Newton observations of PKS 0558-504, a low-redshift quasar $(z=0.137$; $\left.M_{V} \approx-24.5\right)$ identified during the HEAO-1 survey. It has quite narrow Balmer lines $\left(F W H M=1500 \mathrm{~km} \mathrm{~s}^{-1}\right.$, Remillard et al. 1986), which has led to it being routinely described as a Narrow-Line Seyfert 1 (NLS1), although we note that the UV lines, such as Ly $\alpha$, are broader (FWHM $\approx 3800 \mathrm{~km} \mathrm{~s}^{-1}$; Kuraszkiewicz et al. 2000). PKS 0558-504 is unusual in being one of the few radioloud NLS1-type galaxies (Schartel et al. 1996). We find that the X-ray spectrum of PKS 0558-504 is dominated by a strong soft X-ray excess, which may be the highenergy tail of the BBB. The X-ray spectrum is not contaminated by either cold or warm (ionised) absorption features. Soft X-ray narrow emission-lines are also excluded at a high significance level. Thus, we claim to have detected the featureless X-ray continuum emission from a luminous quasar.

\section{XMM-Newton observations}

We present observations of PKS 0558-504 taken with the European Photon Imaging Camera (EPIC) during orbits 42 and 84 (1-2 March 2000 and 24-25 May 2000) of the calibration and performance verification phase of $X M M$ Newton. The EPIC instrument is described in Turner et al. (2001) and Strueder et al. (2001). During both orbits the EPIC MOS cameras were operated in several different observing modes. Here we include only those observations taken with the medium filter, for which the total EPIC MOS exposure times for orbits 42 and 84 are $85.5 \mathrm{ksec}$ and
$53.7 \mathrm{ksec}$ respectively. Only the full-frame mode EPIC PN data for orbit 84 were used, for which the total exposure time is $10.2 \mathrm{ksec}$.

For operational reasons, the EPIC observations in each orbit were divided into several separate exposures of varying lengths. These exposures were reduced using the XMM-Newton SAS (Science Analysis Software) package. The event lists output from the standard EMCHAIN and EPCHAIN scripts were further filtered for each EPIC exposure using the SAS XMMSELECT task. For the MOS exposures only X-ray events corresponding to patterns 012 (similar to $A S C A$ event grades 0-4) were selected. Only pattern 0 (single pixel) events were selected for the PN. Known hot or flickering pixels and electronic noise were rejected using the SAS and the low-energy cutoff was set to $200 \mathrm{eV}$. One of the EPIC MOS-1 images from orbit 42 is shown in Fig. 1.

Source spectra and light curves were extracted from each EPIC exposure using a circular region of diameter 1 arcmin centred on the source position. PKS 0558-504 is by far the brightest X-ray source in the 30 arcmin EPIC field-of-view. The EPIC cameras are subject to non X-ray background events due to particles which can vary significantly during an exposure. Background spectra and light curves for use in later data analysis were derived in a similar way to the source data using an offset region close to the source. We note that the observed countrate for PKS 0558-504 implies little effect due to pile-up and no significant spectral differences were found between observations taken in different MOS window modes. The XSPEC v11.0 software package was used to calibrate the background-subtracted EPIC spectra using the most recent camera response matrices derived from ground-based and in-orbit data. Before further analysis the EPIC spectra were binned to give a minimum of 20 counts per bin.

Reflection Grating Spectrograph (RGS; den Herder et al. 2001) exposures from orbit 84 were also reduced to search for weak/narrow soft X-ray absorption or emission-line features. Three exposures were analysed, two from RGS-1 (each of $25 \mathrm{ksec}$ ) and one from RGS-2 (31 ksec). The RGS data were first processed using the SAS RGSPROC script. The merged RGS event lists were then fed to the SAS EVSELECT and XMMSELECT tasks, and both source and background spectra for each exposure were extracted interactively. These spectra were then calibrated using response matrices derived from the individual RGS exposures using the SAS RMFGEN task.

The Optical Monitor (OM; Mason et al. 2001) imaging data for orbits 42 and 84 were reduced using standard SAS tasks and calibrated with the instrument response information for each filter.

\section{Variability}

No substantial variability was detected within individual EPIC exposures on timescales less than a few minutes, so the data were rebinned and assembled into orbit light curves by combining the individual light curves for each 


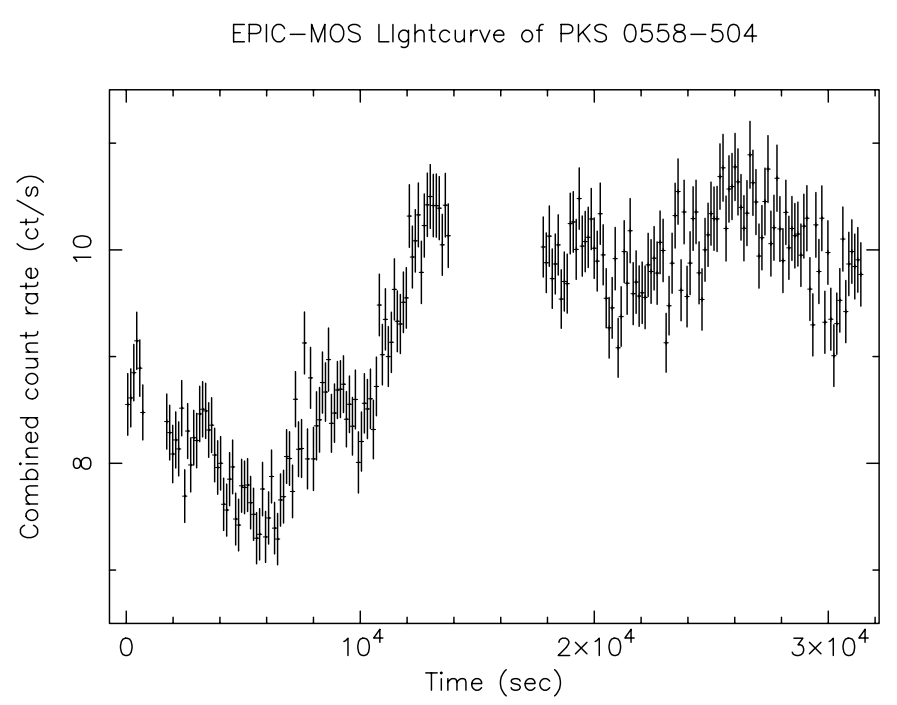

Fig. 2. The EPIC MOS-1 + MOS-2 $0.2-10 \mathrm{keV}$ light curve of PKS $0558-504$ for orbit 84 in 125 s time bins

exposure in order to search for longer duration events. The PKS 0558-504 broad-band EPIC MOS light curve for orbit 84, binned into $125 \mathrm{~s}$ bins, is shown in Fig. 2.

Strong broad-band X-ray flux variability is seen during both orbits, with similar rates of increase and decrease. The largest change in count rate occurs during orbit 84 - an increase of $35 \%$ in 3000 s. Based on the spectral fits discussed below and using $H_{0}=50 \mathrm{~km} \mathrm{~s}^{-1} \mathrm{Mpc}^{-1}$ and $q_{0}=0.5$, this corresponds to a rate of increase in the $0.2-10 \mathrm{keV}$ luminosity of $\Delta L / \Delta t=510^{41} \mathrm{erg} \mathrm{s}^{-2}$. Assuming photon diffusion through a spherical mass of accreting matter in which the opacity is dominated by Thomson scattering, the observed luminosity change implies a minimum efficiency of converting matter into energy of $\eta=(\Delta L / \Delta t) /\left(210^{42}\right)$ (Guilbert et al. 1983). In the simplest interpretation, the change in luminosity observed during orbit 84 therefore implies $\eta=0.25$, an efficiency which exceeds the theoretical maximum for a nonrotating (Schwarzschild) black hole but is consistent with that for a maximally rotating Kerr black hole. Some contribution of beamed X-rays cannot be ruled out, but the shape of the continuum (discussed below) is not consistent with that of a typical beamed source. A tendency for the X-ray continuum to harden slightly when brighter is seen when comparing the data from orbits 42 and 84 . The X-ray variability of PKS $0558-504$ is discussed in more detail in a separate paper (Gliozzi et al. 2001).

During a Ginga observation, the PKS 0558-504 continuum appeared to vary by $67 \%$ in 3 min (implying $\eta \sim 2$; Remillard et al. 1991). Given the high count-rates and well determined background of the XMM-Newton observations, we could have detected such a flare on timescales as short as a few tens of seconds, but none were observed. ROSAT HRI observations also failed to detect such rapid flaring (Gliozzi et al. 2000), but did show luminosity variability rates (allowing for their use of $H_{0}=70 \mathrm{~km} \mathrm{~s}^{-1} \mathrm{Mpc}^{-1}$ ) which imply accretion efficiencies

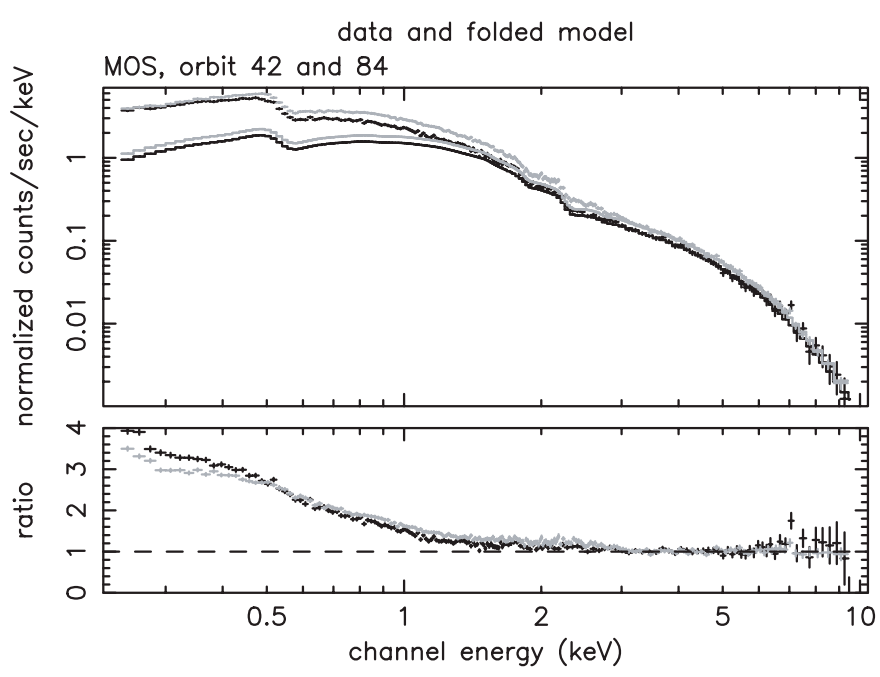

Fig. 3. The EPIC MOS spectra of PKS 0558-504 for orbits 42 and 84. A power-law has been fitted to the $4-10 \mathrm{keV}$ data and extrapolated to lower energies. A broad, strong soft X-ray excess is clearly seen extending to $\sim 3 \mathrm{keV}$

similar to that derived from the XMM-Newton data. Without confirmation using an imaging detector it is impossible to confirm or deny the association of the Ginga flare with intrinsic variability in PKS $0558-504$, so we do not consider the Ginga flare any further in this paper.

\section{Spectral analysis}

Previous analyses of $A S C A$ observations of PKS 0558-504 imply an X-ray spectrum which is softer at lower energies. Fixing the absorbing column density at the Galactic value of $4.410^{20} \mathrm{~cm}^{-2}$, Gliozzi et al. (2000) find $\Gamma=2.99 \pm$ 0.09 over $0.1-2.4 \mathrm{keV}$ from $R O S A T$ data. ASCA data give $\Gamma=2.26 \pm 0.03$ over $0.6-10 \mathrm{keV}$ (Vaughan et al. 1999), whereas the Ginga data reveal a typical AGN power-law spectrum with $\Gamma=1.89_{-0.09}^{+0.04}$ over $2-18 \mathrm{keV}$ (Lawson \& Turner 1997). Similarly, Comastri (2000) finds $\Gamma=1.97 \pm$ 0.04 over $2-10 \mathrm{keV}$ from BeppoSAX data.

As noted above, no large ( $\gtrsim 10 \%)$ changes in spectral shape were observed during each orbit. Thus, to improve the signal-to-noise ratio the MOS data were combined to form a mean spectrum for each orbit before detailed spectral analysis.

The background-subtracted, summed MOS observations provide the highest $\mathrm{S} / \mathrm{N}$ data for PKS 0558-504 to date. These spectra are very poorly fitted by a single power-law $\left(\chi_{\nu}^{2}=2.75\right)$ due to the presence of a large, broad soft X-ray excess which dominates the X-ray emission from PKS 0558-504 in the XMM-Newton bandpass. To illustrate this feature, Fig. 3 shows the MOS spectra for each orbit with a power-law fitted to the $4-10 \mathrm{keV}$ band, allowing for Galactic absorption (fit 1 in Table 1 - all the fits are summarised in Table 1). The powerlaw is fitted above $4 \mathrm{keV}$ as the soft excess appears to extend to $\sim 3 \mathrm{keV}$. The PN spectrum for orbit 84 is 
The Soft Excess in PKS 0558-504

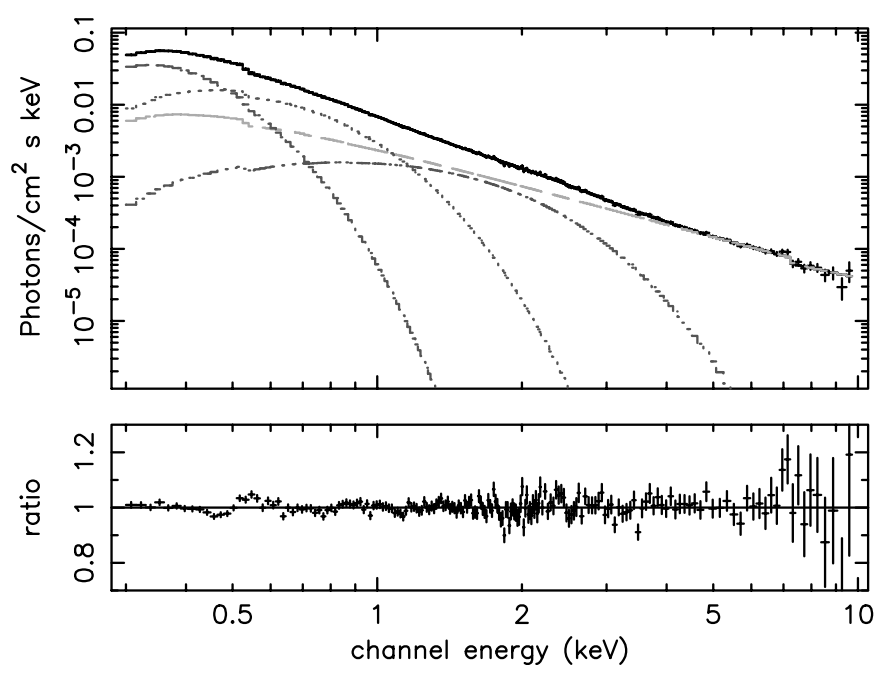

Fig. 4. The multiple blackbody plus power-law fit to the combined orbit 42 and 84 MOS data

consistent with the MOS allowing for the current calibration uncertainties.

No evidence is found for a significant change in $\mathrm{X}$-ray spectral shape between the two orbits, but there is a small decrease in the mean X-ray flux of $\approx 18 \%$ from orbit 42 to 84 . The OM data show that the UV flux also decreased by $\approx 10 \%$ from orbit 42 to 84 . For orbit 42 the $0.2-2.0 \mathrm{keV}$ and $2-10 \mathrm{keV}$ fluxes are $2.4410^{-11}$ and $1.0110^{-11} \mathrm{erg} \mathrm{cm}^{-2} \mathrm{~s}^{-1}$ respectively, corresponding to luminosities of $3.710^{45}$ and $0.9810^{45} \mathrm{erg} \mathrm{s}^{-1}$.

The soft X-ray excess in PKS 0558-504 is far too broad to be fitted by a single temperature blackbody. To parameterise the spectra with a relatively simple model, we fitted a combination of blackbodies and a power-law. At least three blackbodies are required of 80,175 and $475 \mathrm{eV}$, to give an acceptable fit $\left(\chi_{\nu}^{2}=1.2\right.$; fit 2 in Table 1). Other than the relative normalisation (consistent with the small overall change in flux), the derived power-law indices and blackbody temperatures required to fit the mean spectra from orbits 42 and 84 are statistically consistent. Therefore, we combined the entire MOS data before proceeding. The multiple blackbody and power-law fit to the combined MOS data is shown in Fig. 4. The highenergy power-law slope in the multi-component model is consistent with the $\Gamma=1.9$ commonly seen in radio-quiet AGN.

No iron fluorescence line is detected from either neutral or ionised material. For the combined MOS data, the upper limits to the $\mathrm{EW}$ of a $6.4 \mathrm{keV}$ line are $<10 \mathrm{eV}$ for a narrow line $(\sigma=10 \mathrm{eV})$ and $<90 \mathrm{eV}$ for a broad line $(\sigma=300 \mathrm{eV})$. For a narrow ionised line at $6.7 \mathrm{keV}$, the upper limit is $42 \mathrm{eV}$. In addition, no evidence is found in the EPIC or RGS spectra for any intrinsic cold or warm absorption. The O VII or O viII edge optical depth limits are $\tau<0.1$. The upper limit for an ionised iron edge is $\tau<0.2$. We note that an apparent feature in Figs. 3, 4 and 6 at $\approx 7 \mathrm{keV}$ (which is the wrong energy for an iron
Opt-UV-Xray SED of PKS 0558-504

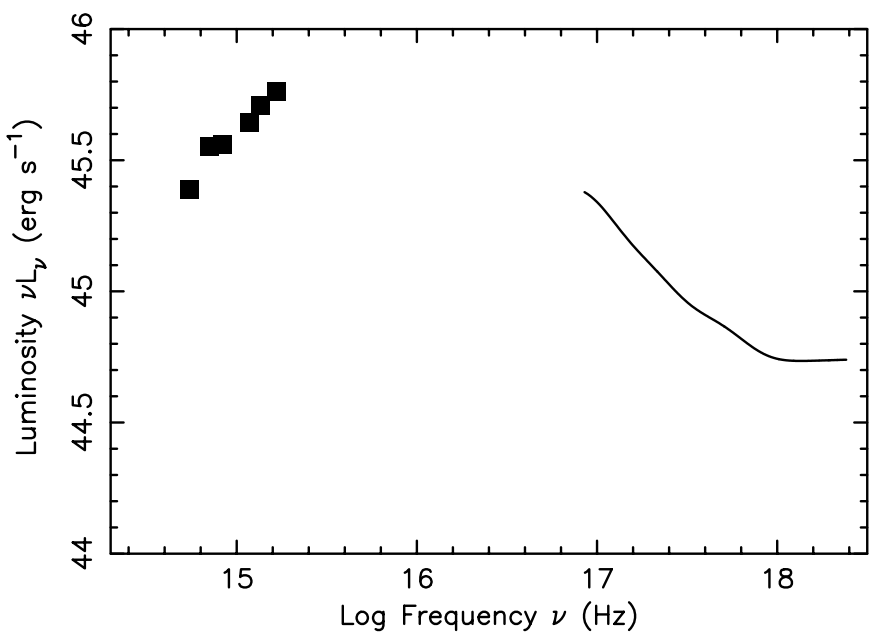

Fig. 5. The broad-band spectral energy distribution of PKS 0558-504 from OM and EPIC data

line in the QSO rest-frame) is due to noise in some of the MOS spectra that went to form the mean. This feature is not present in the PN spectra. Overall, PKS 0558-504 is a powerful quasar in the X-ray band, with a $0.25-10 \mathrm{keV}$ (unabsorbed) luminosity of $4.510^{45} \mathrm{erg} \mathrm{s}^{-1}$ mostly emitted below $3 \mathrm{keV}$. The lack of spectral features strongly implies that we are seeing the bare quasar continuum emission.

\section{Discussion}

The multiple-blackbody plus power-law model discussed above provides an acceptable fit to the EPIC spectra, but has no physical basis. To examine the broad-band spectral energy distribution (SED) of PKS 0558-504 the dereddened optical and UV data from the OM (assuming Galactic $E(B-V)=0.044)$ together with the X-ray continuum derived from the combined MOS data (using the multiple blackbody model) are shown in Fig. 5 .

The SED of PKS 0558-504 clearly shows an opti$\mathrm{cal} / \mathrm{UV} \mathrm{BBB}$, and the XMM-Newton data suggest this feature extends into the X-ray band. To power an object as luminous as PKS 0558-504 ( $\left.L_{\mathrm{bol}} \sim 10^{46} \mathrm{erg} \mathrm{s}^{-1}\right)$ would require a black hole of mass $M_{\mathrm{BH}} \sim 10^{8} M_{\odot}$ accreting at the Eddington rate. Based on accretion disc models (e.g. Czerny \& Elvis 1987), the thermal spectrum of such a system could explain the optical/UV portion of the BBB but would be unable to produce the broad, soft X-ray excess or the high-energy power-law-like emission.

One model suggested to explain the steep X-ray spectra of extreme soft X-ray sources, such as NLS1s, is that these source accrete at an unusually high rate leading to a large soft photon flux. These photons then Compton cool the hard photon source, generated in a hot accretion disc corona, resulting in a steeper X-ray spectrum (Pounds et al. 1995). The temperature, geometry and covering factor of disc coronae are poorly defined from theory, 
Table 1. Fits to $X M M-N e w t o n$ data in the $0.2-10 \mathrm{keV}$ band. ${ }^{\mathrm{a}}$ Powerlaw index for fit to $4.0-10 \mathrm{keV}$. ${ }^{\mathrm{b}}$ Electron temperature of thermal source $(\mathrm{eV}) .{ }^{\mathrm{c}}$ Electron temperature of Comptonizing electrons $(\mathrm{keV}) .{ }^{\mathrm{d}}$ Optical depth

\begin{tabular}{lllccr}
\hline Rev/Camera & Fit & Model & $\Gamma /$ photon $k T$ & BB or electron temp $(k T) /$ optical depth & $\chi^{2} /$ dof \\
\hline $42 / \mathrm{MOS}$ & 1 & $\mathrm{PL}^{\mathrm{a}}$ & $2.12 \pm 0.15$ & - & $241 / 243$ \\
$42 / \mathrm{MOS}$ & 2 & $\mathrm{PL}+3 \times \mathrm{BB}$ & $2.03 \pm 0.09$ & $82 \pm 5,173 \pm 10,469 \pm 18$ & $608 / 489$ \\
$84 / \mathrm{MOS}$ & 1 & $\mathrm{PL}^{\mathrm{a}}$ & $1.99 \pm 0.15$ & - & $276 / 258$ \\
$84 / \mathrm{MOS}$ & 2 & $\mathrm{PL}+3 \times \mathrm{BB}$ & $1.97 \pm 0.12$ & $74 \pm 5,165 \pm 11,454 \pm 35$ & $622 / 493$ \\
$84 / \mathrm{PN}$ & 1 & $\mathrm{PL}$ & $1.94 \pm 0.17$ & - & $188 / 192$ \\
$84 / \mathrm{PN}$ & 2 & $\mathrm{PL}+3 \times \mathrm{BB}$ & $1.84 \pm 0.05$ & $89 \pm 5,189 \pm 14,560 \pm 50$ & $1009 / 798$ \\
$42+84 \mathrm{MOS}$ & 1 & PL & $2.07 \pm 0.12$ & - & $319 / 317$ \\
$42+84 \mathrm{MOS}$ & 2 & $\mathrm{PL}+3 \times \mathrm{BB}$ & $1.97 \pm 0.10$ & $80 \pm 3,175 \pm 6,475 \pm 15$ & $721 / 556$ \\
$42+84 \mathrm{MOS}$ & 3 & CompTT & $59 \pm 8^{\mathrm{b}}$ & $4.0_{-1.5}^{+4.5 \mathrm{c}}, 2.5_{-1.1}^{+0.8 \mathrm{~d}}$ & $780 / 559$ \\
& & & $59 \pm 8^{\mathrm{b}}$ & $55_{-35}^{+65 \mathrm{c}}, 0.7 \pm 0.4^{\mathrm{d}}$ & \\
\hline
\end{tabular}

Comptonized Spectrum of PKS 0558-504

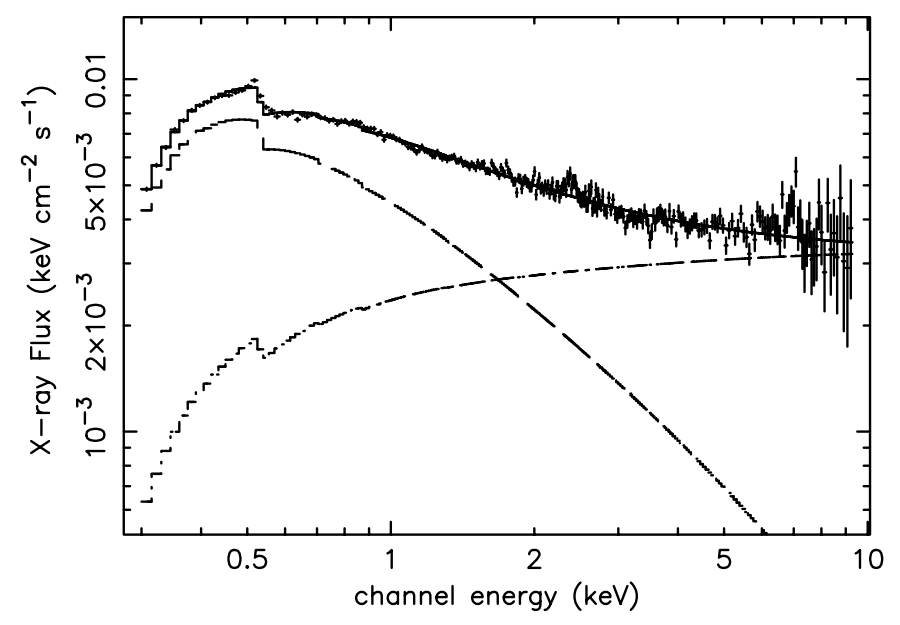

Fig. 6. The Comptonization model fit to the combined orbit 42 and 84 MOS data plotted in $\nu f_{\nu}$ space

but multiple-temperatures are likely to be required due to vertical temperature stratification.

To explore Comptonization models, the results of fitting the XsPec model COMPTT to the combined orbit 42 and 84 data are given in Table 1 (fit 3) and shown in Fig. 6. A model involving two electron populations, both scattering photons from a single temperature thermal source, provides a good fit to the mean X-ray spectrum. Allowing the thermal source temperature to vary independently does not produce a significantly better fit. The temperature and luminosity of the soft photon source are consistent with that of a thermal accretion disc spectrum for a $10^{8} M_{\odot}$ black hole accreting at close to the Eddington rate. The Comptonizing electron temperatures of 4 and $55 \mathrm{keV}$ are at the low end of the expected temperature range (Haardt \& Maraschi 1993), possibly due to the large soft photon flux Compton cooling the corona. We note that the exact values of the parameters for the two electron populations in the comptt model are quite poorly constrained. This is because increasing the optical depth slightly can harden the spectrum in a similar way to adding a higher temperature electron population, thus giving a variety of two-component models that adequately fit the data.

We note that, as suggested by the power-law and multiple blackbody fits, the high-energy tail of the Comptonized spectrum has a shape consistent with that of "normal" broad-line AGN power-laws $(\Gamma \sim 1.9)$, and is significantly harder than that derived from the $A S C A$ data. These differences are presumably due to the inability of the $A S C A$ data to delineate the tail of the soft $\mathrm{X}$-ray excess which extends to around $3 \mathrm{keV}$ in this source. The observed mean slope of the hard power-law in AGN $(\Gamma=1.9)$ is predicted from models in which feedback occurs between soft photons produced by hard X-ray heating Compton cool a moderate optical-depth corona (Haardt \& Maraschi 1993). Such a model cannot explain the bulk of the soft X-ray luminosity in PKS 0558-504 as it dominates the luminosity, but may produce the residual hard power-law emission.

The reduced chi-squared value for the Comptonized model $\left(\chi_{\nu}^{2}=1.3\right)$ shows that the Comptonization model fit leaves some spectral curvature, mainly above a few $\mathrm{keV}$. Some of this imperfection is due to remaining calibration uncertainties around features such as detector Gold edge, but most of it is due to the Comptonization model simply being too simplistic to adequately fit such high-quality data. Some of the remaining spectral curvature can be reduced by including an additional Comptonizing component (i.e. hotter electrons) but this component does not formally improve the fit in terms of the reduced chi-squared presumably because the XMM-Newton highenergy cutoff is too low to adequately constrain a very-hot electron population.

An alternative explanation for a high-energy "powerlaw-like" component is a small amount of reflection from an ionised disc. The disc is likely to be ionised given the luminosity of the source. Indeed, the absence of any strong iron $\mathrm{K} \alpha$ emission line or iron edge at high energies and the lack of absorption features due to 
moderately ionised material at low energies suggests that any X-ray heated disc will have a substantial "skin" which is fully ionised and effectively acting as a "mirror". This has been suggested for high accretion rate sources in the context of a magnetic-flare model for X-ray variability (Nayakshin 2000). The small iron $\mathrm{K} \alpha$ emission line and iron edge strengths predicted by the magnetic-flare model, assuming $\Gamma \sim 2$, are consistent with the upper limits from the XMM-Newton spectra (Nayakshin, private communication). What is less clear is if a fully ionised corona can be maintained in a source like PKS 0558-504 with a large soft photon flux if those photons are generated close to the magnetic-flares. Clearly the XMM-Newton data for PKS 0558-504 demand comparison with more detailed accretion disc models.

\section{Conclusions}

The XMM-Newton X-ray spectrum of PKS 0558-504 is dominated by a large, variable soft excess. The fastest observed variability implies a minimum efficiency of converting matter to energy of $\eta=0.25$, suggesting accretion onto a Kerr black hole. Combining the X-ray data with simultaneous OM optical and UV data reveal a large big blue bump; too broad to be due purely to a thermal accretion disc spectrum. The X-ray spectrum can be fitted by a Comptonization model in which thermal photons, presumably from the disc, are scattered by a hot corona. At least two electron temperatures are required. Overall, PKS 0558-504 can be explained by an accretion disc running at a high accretion rate producing copious amounts of soft EUV photons which are then Compton cooling a hot disc corona.

Acknowledgements. Based on observations obtained with the $X M M-N e w t o n$, and ESA science mission with instruments and contributions diectly funded by ESA member states and the USA (NASA). EPIC was developed by the EPIC Consortium led by the Principal Investigator, Dr. M. J. L. Turner. The consortium comprises the following Institutes: University of Leicester, University of Birmingham, (UK); CEA/Saclay, IAS Orsay, CESR Toulouse, (France); IAAP Tuebingen, MPE Garching, (Germany); IFC Milan, ITESRE Bologna, IAUP Palermo, (Italy). EPIC is funded by: PPARC, CEA, CNES, DLR and ASI. We thank the EPIC and SSC teams for much help and advice, particularly Steve Sembay, Gareth Griffiths,
Richard West and Ian Stewart. We also thank Kim Page for help with data reduction.

\section{References}

Barvainis, R. 1993, ApJ, 412, 513

Comastri, A. 2000, New Astron. Rev., in press

Czerny, B., \& Elvis, M. 1987, ApJ, 321, 305

den Herder, J. W., Brinkmann, A. C., Kahn, S. M., et al. 2001, A\&A, 365, L7

Elvis, M., Wilkes, B. J., McDowell, J. C., et al. 1994, ApJS, 95,1

George, I. M., \& Fabian, A. C. 1991, MNRAS, 249, 352

Gliozzi, M., Boller, Th., Brinkman, W., \& Brandt, W. N. 2000, $\mathrm{A} \& \mathrm{~A}$, in press

Gliozzi, M., Brinkmann, W., O'Brien, P. T., et al. 2001, A\&A, 365, L128

Guilbert, P. W., Fabian, A. C., \& Rees, M. J. 1983, MNRAS, 205, 593

Haardt, F., \& Maraschi, L. 1993, ApJ, 413, 507

Kuraszkiewicz, J., Wilkes, B. J., Czerny, B., \& Mathur, S. 2000, ApJ, in press

Laor, A., Fiore, F., Elvis, M., Wilkes, B. J., \& McDowell, J. C. 1997, ApJ, 477, 93

Lawson, A. J., \& Turner, M. J. L. 1997, MNRAS, 288, 920

Mason, K. O., Breeveld, A., Much, R., et al. 2001, A\&A, 365, L36

Nayakshin, S. 2000, ApJ, 540, L37

Pounds, K. A., Done, C., \& Osborne, J. 1995, MNRAS, 277, L5

Pounds, K. A., Nandra, K., Stewart, G. C., George, I. M., \& Fabian, A. C. 1990, Nat, 344, 132

Remillard, R. A., Bradt, H. V., Buckley, D. A. H., et al. 1986, ApJ, 301, 742

Remillard, R. A., Grossen, B., Bradt, H. V., et al. 1991, Nat, 350,589

Ross, R. R., \& Fabian, A. C. 1993, MNRAS, 261, 74

Schartel, N., Walter, R., Fink, H. H., \& Trümper, J. 1996, A\&A, 307, 33

Shields, G. A. 1979, Nat, 272, 706

Siemiginowska, A., Kuhn, O., Elvis, M., et al. 1995, ApJ, 454, 77

Strüder, L., Briel U., Dennerl, K., et al. 2001, A\&A, 365, L18

Turner, M. J. L., Abbey, A., Arnaud, M., et al. 2001, A\&A, 365, L27

Turner, T. J., \& Pounds, K. A. 1989, MNRAS, 240, 833

Vaughan, S., Reeves, J., Warwick, R., \& Edelson, R. 1999, MNRAS, 309, 113

Walter, R., Orr, A., Courvoisier, T. J.-L., et al. 1994, A\&A, 285,119 